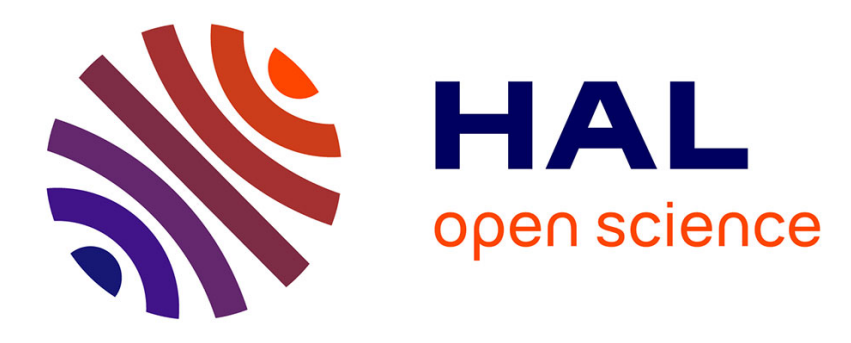

\title{
Usage des indicateurs de capital humain et pilotage de la performance
}

Stephen Gates, Pascal Langevin

\section{To cite this version:}

Stephen Gates, Pascal Langevin. Usage des indicateurs de capital humain et pilotage de la performance. Revue Française de Gestion, 2010, 8 (207), pp.125-138. 10.3166/rfg.207.125-138 . hal00796945

\section{HAL Id: hal-00796945 \\ https://hal-audencia.archives-ouvertes.fr/hal-00796945}

Submitted on 13 Sep 2013

HAL is a multi-disciplinary open access archive for the deposit and dissemination of scientific research documents, whether they are published or not. The documents may come from teaching and research institutions in France or abroad, or from public or private research centers.
L'archive ouverte pluridisciplinaire HAL, est destinée au dépôt et à la diffusion de documents scientifiques de niveau recherche, publiés ou non, émanant des établissements d'enseignement et de recherche français ou étrangers, des laboratoires publics ou privés. 
DOSSIER

\section{STEPHEN GATES}

Audencia Nantes École de management

\section{PASCAL LANGEVIN}

EM Lyon

\section{Usage des indicateurs de capital humain et pilotage de la performance}

Cet article présente les résultats d'une étude empirique sur I'usage des indicateurs de capital humain. Outre des éléments descriptifs sur la manière dont les indicateurs sont diffusés au sein de l'entreprise, les résultats montrent également que la performance est positivement associée à la mise en place de ces indicateurs, mais que cette mise en place n'a pas encore atteint un niveau avancé. 
$\mathrm{D}$ epuis une vingtaine d'années, la littérature en gestion reconnaît le rôle fondamental du capital humain dans l'acquisition d'un avantage concurrentiel et dans la création de valeur (Hamel and Prahalad, 1994; Lev, 2001). Le capital humain fait référence aux connaissances, compétences, expériences, ainsi qu'aux motivations et attitudes des salariés d'une organisation (Davenport, 1999). Il est généralement considéré comme la composante du capital intellectuel ${ }^{1}$ qui a la plus grande valeur (Backhuijs et al., 1999). Compte tenu de ce rôle critique du capital humain, la question se pose de son pilotage et - si on accepte l'adage que n'est gérable que ce qui est mesurable - de sa mesure (Marr et al., 2003).

Les études constatant l'insuffisance des indicateurs financiers pour gérer efficacement l'entreprise à long terme (Eccles, 1991; Ittner and Larcker, 1998) suggèrent aux organisations de piloter les inducteurs de performance aux niveaux opérationnels les plus en amont possible. Cela a conduit à la proposition de cadres conceptuels utilisant des indicateurs non financiers articulés selon des relations de cause à effets. Les indicateurs de capital humain (ICH) comptent parmi ces indicateurs non financiers de la performance.

Cette mesure du capital humain devrait donc être au cœur des préoccupations de nombreuses fonctions, en particulier, les directions qui souhaitent disposer des compétences clés pour atteindre leurs objectifs stratégiques, les responsables des ressources humaines en charge du développement de ce capital humain et les contrôleurs, experts de la mesure de la performance.

Pour autant, si les indicateurs de capital humain ont effectivement fait l'objet d'un intérêt croissant (Becker et al., 2001; Boudreau et Ramstad, 2003; Fitz-Enz, 1995; Huselid et al., 2005), ils semblent déconnectés de la stratégie. Selon une étude publiée par le Conference Board, seulement $12 \%$ des répondants déclaraient utiliser des indicateurs humains pour piloter leur performance stratégique (Gates, 2004).

L'objectif de cette étude est donc d'explorer plus avant, à partir d'une étude empirique, quantitative et qualitative, les pratiques des entreprises en termes d'utilisation des indicateurs de capital humain et leur relation avec la stratégie et la performance.

Dans la première partie, nous présentons les résultats qui montrent que la performance est effectivement liée à la mise en place d'indicateurs de capital humain mais, aussi, que peu d'entreprises ont atteint un niveau élevé dans cette mise en place. Dans la deuxième partie, nous nous intéressons aux caractéristiques de ces indicateurs et à la manière dont ils sont diffusés dans l'entreprise. Nous montrons dans la troisième partie le lien entre stratégie et indicateurs. Enfin, la cinquième partie présente le rôle joué par les responsables RH et les difficultés rencontrées dans la mise en place de ces indicateurs de capital humain.

1. Bien que la définition du capital intellectuel varie (Edvinsson and Malone, 1997; OECD, 1999), trois composantes sont généralement retenues : le capital humain, le capital structurel qui correspond aux structures, procédures, routines, bases de données qui permettent à une organisation de codifier, d'organiser et de diffuser en interne les connaissances provenant du capital humain et le capital relationnel qui intègre la connaissance des relations de l'organisation avec ses partenaires externes, tels que ses clients, fournisseurs ou collectivités (Edvinsson et Malone, 1997). Cet article s'intéresse uniquement au capital humain. 


\section{RECUEIL DES DONNÉES}

Les résultats présentés dans cet article sont issus d'un projet de recherche mené en collaboration avec le Conference Board, organisation dont l'objet est de permettre à ses membres, experts des différentes fonctions de l'entreprise, d'échanger et progresser sur leurs pratiques, aux travers de conférences, études, publications, etc.

Létude repose à la fois sur une approche par questionnaires et sur une approche par entretiens. L'un des auteurs de cet article a animé deux groupes de travail (au total 51 experts de la fonction $\mathrm{RH}$ ), réunis (à trois reprises chacun) pour échanger sur la mesure du capital humain. Six participants, choisis pour leur niveau plus avancé de mise en place d'indicateurs de capital humain, ont été interviewés de façon plus approfondie et ont contribué à la réalisation du questionnaire.

Après prétest, ce questionnaire a été adressé aux 51 participants des groupes de travail, puis par internet auprès de 400 membres de la section RH du Conference Board. Au total, l'étude quantitative a été réalisée sur les 104 réponses obtenues (taux de réponse de $23 \%$ ). Les tests statistiques n'ont montré aucune différence entre les réponses provenant de ces deux groupes.

Dans les entreprises de l'échantillon, $77 \%$ ont leur siège en Amérique du Nord, $16 \%$ en Europe et $7 \%$ dans le reste du monde. $46 \%$ ont un chiffre d'affaires supérieur à 5 milliards de dollars; $30 \%$ entre 1 et 5 milliards et $24 \%$ inférieurs à 1 milliard. $24 \%$ emploient plus de 50000 salariés, $44 \%$ entre 5000 et $50000,32 \%$ moins de 5000 . Enfin, $26 \%$ sont dans le secteur industriel, $35 \%$ dans les services non financiers, $21 \%$ dans les services financiers, $8 \%$ dans les secteurs de l'énergie, et $10 \%$ dans d'autres secteurs.

\section{Mise en place des indicateurs de capital humain et performance}

De nombreuses études analysent le lien entre capital humain et performance (voir Ashton, 2005, pour une revue). Si le capital humain joue un rôle fondamental dans la création de valeur, l'utilisation d'indicateurs de capital humain pertinents peut contribuer fortement à l'atteinte des objectifs stratégiques de l'entreprise. En effet, les managers peuvent utiliser ces indicateurs pour identifier et mieux gérer les compétences clés. Une bonne performance au niveau du capital humain augmente les chances d'avoir une bonne performance au niveau de l'organisation dans son ensemble. Les différentes formes de carte stratégique ou de tableaux de bord, tels que la Balanced Scorecard, avec sa perspective « apprentissage et développement » (Kaplan et Norton, 1996) ou le schéma de valeur de Skandia et sa formulation dans le Navigator (Edvinsson, 1997) sont des exemples frappants d'approches où les indicateurs de capital humain sont considérés comme critiques pour atteindre les objectifs stratégiques. On peut donc s'attendre à ce que plus une organisation est avancée dans la mise en place d'indicateurs de capital humain, plus elle est performante. La mise en place de ces 
indicateurs est un processus qui passe par plusieurs étapes. Boudreau et Ramstad (2003) proposent trois catégories d'indicateurs qui correspondent aux différents niveaux d'avancement dans leur mise en place: la première étape concerne les indicateurs d'efficience qui se concentrent essentiellement sur les coûts et la productivité des activités de GRH. Puis viennent les indicateurs d'efficacité qui s'intéressent à l'efficacité des programmes GRH à développer les compétences, la motivation et l'attitude des salariés. Enfin, le niveau le plus avancé correspond aux indicateurs d'impact qui mesurent l'effet des processus et programmes de GRH sur la performance de l'organisation.

Les résultats de notre étude montrent que la performance est associée positivement au niveau de mise en place des indicateurs de capital humain ${ }^{2}$. Des citations tirées de nos entretiens confirment que la mesure du capital humain facilite la prise de décision.
« Le nouveau directeur prévoit d'utiliser des indicateurs de capital humain dans un tableau de bord pour commencer chaque réunion mensuelle avec les managers $\mathrm{RH}$ des divisions. Ce tableau de bord sera utilisé comme un outil de diagnostic permettant des décisions informées. » «Une fois intégrés dans les tableaux de bord des managers, les indicateurs de capital humain sont entrés dans leur champ d'attention et les résultats se sont améliorés. » «Au fur et à mesure que les responsables de divisions sont devenus plus à l'aise avec les indicateurs de capital humain, ils ont commencé à poser des questions plus pertinentes et à être plus ouverts à des suggestions de solutions basées sur ces informations. ।

Ce résultat doit néanmoins être regardé avec précaution, car beaucoup d'entreprises dans notre étude n'ont pas atteint un niveau avancé de mise en place d'indicateurs de capital humain. Le tableau 1 montre en effet

Tableau 1 - Niveau de mise en place des ICH

(\% des répondants)

\begin{tabular}{|l|c|}
\hline Aucun indicateur de capital humain & $2,0 \%$ \\
\hline Indicateurs de capital humain en cours de mise en place & $27,7 \%$ \\
\hline Indicateurs d'efficience (temps et coût) en place & $27,7 \%$ \\
\hline Indicateurs d'efficience et d'efficacité (compétences, motivation, performances) en place & $35,7 \%$ \\
\hline $\begin{array}{l}\text { Indicateurs d'efficience, d'efficacité et d'impact (effets des processus sur la performance) } \\
\text { en place }\end{array}$ & $6,9 \%$ \\
\hline Total & $100 \%$ \\
\hline
\end{tabular}

$\mu=3,18 ; \sigma=0,98 ; N=101$

2. $\left(\mathrm{R}^{2}=0,280, \mathrm{p}<0,01\right)$. Nous avons conçu une question à cinq points basée sur les catégories de Boudreau et Ramstad (2003) pour mesurer le niveau de mise en place des ICH (voir les items du tableau 1). La performance a été, quant à elle, mesurée à l'aide d'une question à huit items développée par Huang (2001). Une analyse en composantes principales a conduit à éliminer deux items. Les six items restant étaient tous représentés par une seule composante, expliquant $76 \%$ de la variance, avec un alpha de Cronbach de 0,94. 
que seulement $43 \%$ de l'échantillon ont atteint les deux derniers niveaux, ceux pour lesquels le lien entre indicateurs de capital humain et performance peut vraiment devenir efficace. Un certain nombre de raisons à ce faible développement seront analysées plus loin.

Notre étude s'est également intéressée aux caractéristiques de ces indicateurs et à leur diffusion au sein de l'entreprise.

\section{Reporting des indicateurs de capital humain et tableaux de bord}

La littérature en contrôle indique que pour constituer un outil d'aide à la gestion du capital humain, les indicateurs doivent être communiqués de façon périodique, sous une forme appropriée et aux managers adéquats.

On constate tout d'abord que la périodicité $\mathrm{du}$ reporting aux senior-managers se fait, pour $82 \%$ des entreprises répondantes $(\mathrm{N}=99)$, sur une base au moins mensuelle et/ou trimestrielle, les $18 \%$ restant utilisant uniquement une fréquence semestrielle et/ou annuelle. Cette fréquence peut permettre de prendre les actions correctives nécessaires assez rapidement.

Nos résultats indiquent également une assez grande variété dans le nombre d'indicateurs utilisés dans le reporting aux senior-managers (figure 1).

Mais la majorité des répondants $(75 \%)$ utilisent au plus dix indicateurs et seulement $9 \%$ en utilisent plus de vingt. Cela est cohérent avec l'idée que les managers peuvent avoir des difficultés à se concentrer sur un nombre trop important d'indicateurs.

La communication de ces indicateurs de capital humain s'adresse, pour $38 \%$ des répondants $(\mathrm{N}=97)$ uniquement au topmanagement ou au comité de direction. Les managers opérationnels sont donc destinataires de ces indicateurs dans $62 \%$ des cas. Ce résultat laisse donc penser qu'une grande majorité d'entreprises considère que le capital humain n'est pas uniquement une problématique de dirigeants, mais qu'il

Figure 1 - Nombre d'ICH reportés aux seniors-managers

( $\%$ des répondants, $\mathrm{N}=85$ )

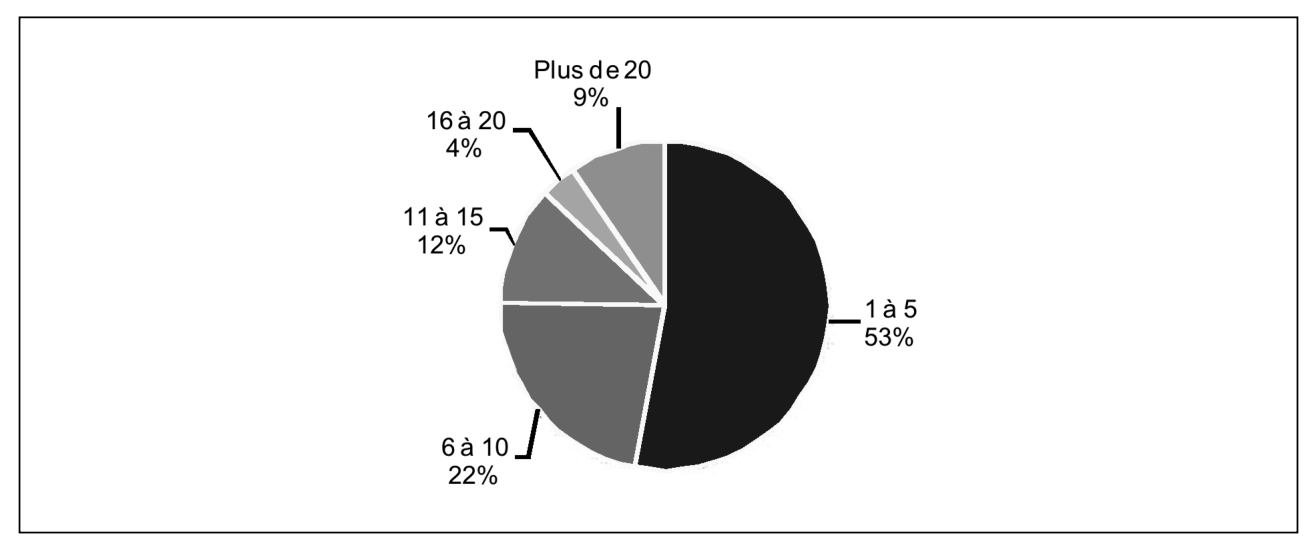


constitue un élément de la gestion opérationnelle.

La littérature insiste sur l'importance d'intégrer les indicateurs de mesure de performance au sein de tableaux de bord. Par exemple, Huselid et al. (2005) défendent l'idée qu'un tableau de bord de la force de travail (Workforce scorecard) est un moyen pour les managers de gérer stratégiquement leurs collaborateurs. Il vient s'ajouter au tableau de bord général de l'entreprise ainsi qu'aux tableaux de bord de suivi des activités de la fonction RH. $66 \%$ de nos répondants déclarent que les indicateurs de capital humain sont utilisés au sein d'un tableau de bord (figure 2). Toutefois, seulement $28 \%$ considèrent ce tableau de bord comme réellement efficace. Clairement, même quand les indicateurs de capital humain sont intégrés dans un tableau de bord, cela ne garantit pas qu'ils soient appliqués avec succès.

Une hypothèse pouvant expliquer ce résultat serait que les managers ne sont pas incités à porter attention à ces indicateurs, en particulier par l'utilisation de systèmes de bonus. En effet, Gates (2003) observe que la prise en compte de la gestion du capital humain dans des plans de bonus est corrélée avec le succès de l'alignement de certains indicateurs de capital humain (turnover, leadership, santé et sécurité) avec la stratégie. Qu'observe notre étude à ce sujet?

Dans notre échantillon, $57 \%$ des répondants intègrent des indicateurs de capital humain dans le calcul des bonus. Par ailleurs, $47 \%$ indiquent que ces indicateurs comptent pour au moins $20 \%$ dans le calcul du bonus (figure 3 ).

À ce niveau de pondération, on pourrait penser que les managers sont incités à porter sérieusement attention aux activités et indicateurs de capital humain, comme l'indique la remarque de l'un des répondants :

«Nous pouvons responsabiliser les divisions davantage sur leur ICH (turnover, transferts, etc.) afin de les forcer à utiliser ces données plus régulièrement. "

Figure 2 - Utilisation et efficacité des tableaux de bord intégrant des ICH

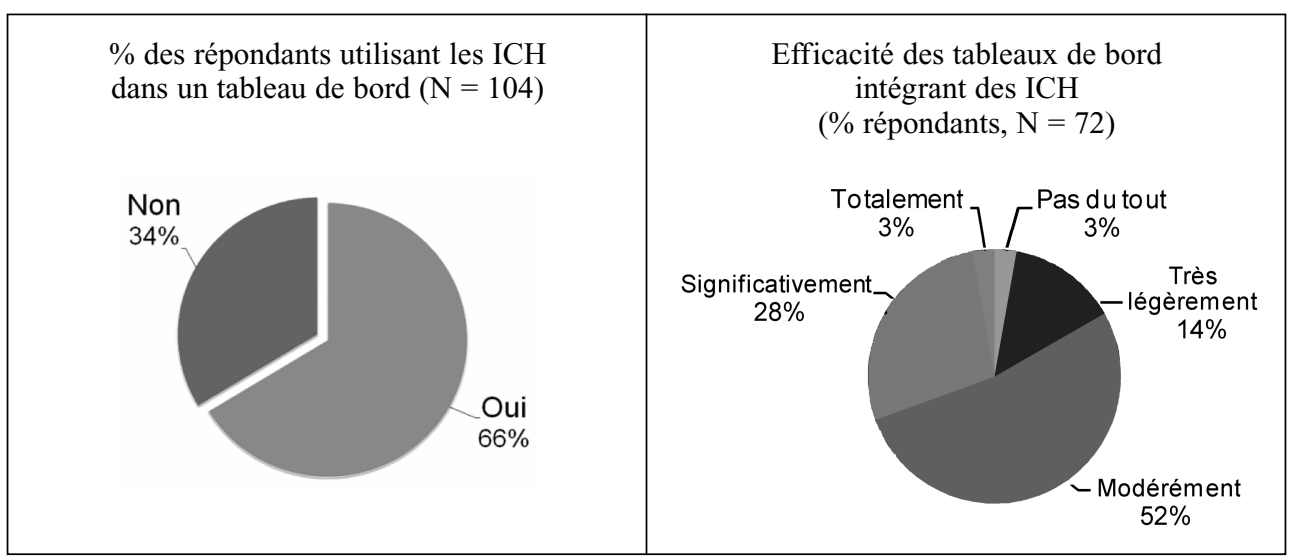


Figure 3 - Prise en compte des ICH dans le calcul des bonus

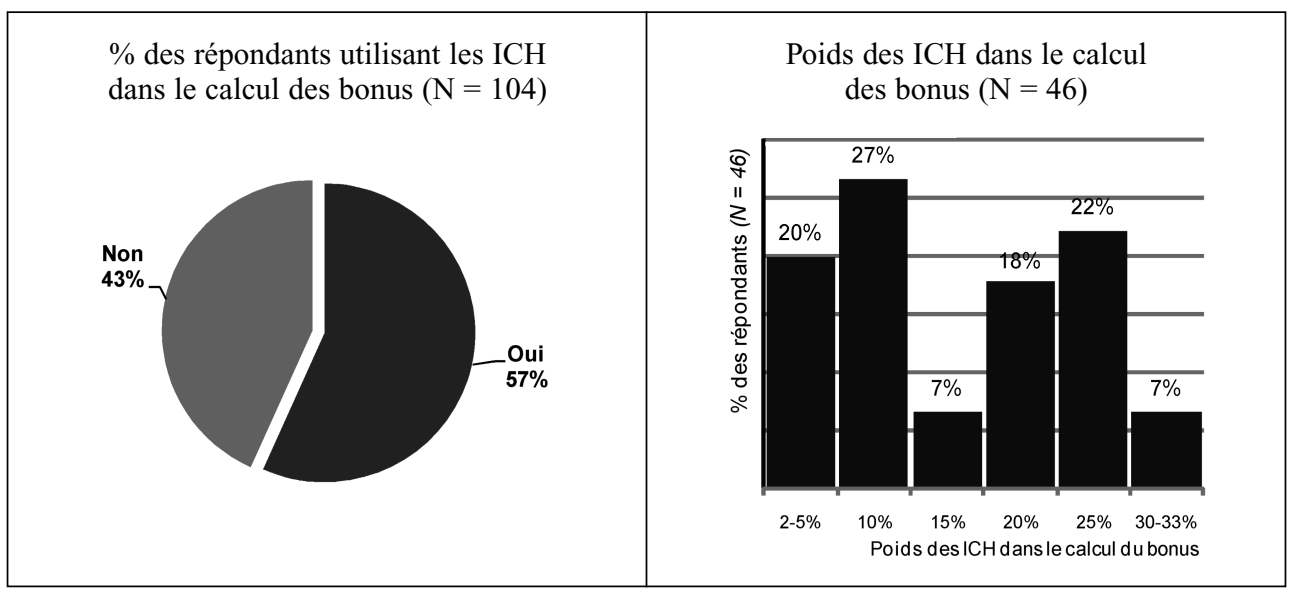

Figure 4 - Degré d'alignement des bonus basés sur les ICH avec les objectifs ( $\%$ des répondants, $\mathrm{N}=58$ )

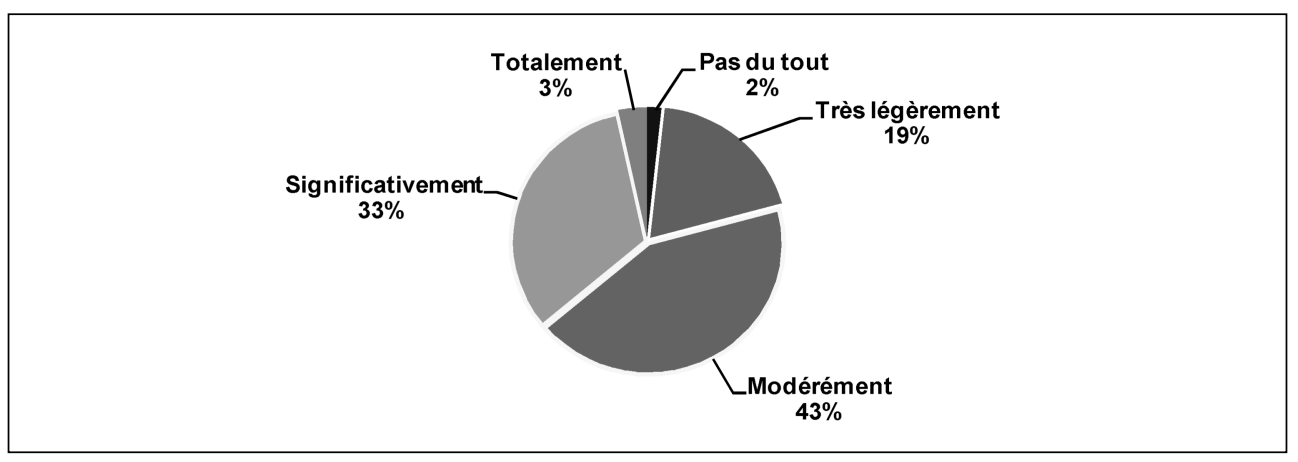

Pourtant, seulement $36 \%$ des répondants considèrent que les indicateurs de capital humain utilisés dans ces bonus sont en ligne avec les objectifs (figure 4). Ce faible alignement peut jeter des doutes, dans notre échantillon, quant à la capacité des indicateurs de capital humain pour aider les managers à atteindre leurs objectifs. On constate effectivement que $15 \%$ seulement des managers utilisent les indicateurs de capital humain de façon importante pour atteindre leurs objectifs (figure 5).

Une explication possible de ce résultat pourrait se situer dans la faible adaptation des indicateurs de capital humain aux caractéristiques de chaque division. La figure 6 indique en effet que $13 \%$ seulement des répondants déclarent avoir des indicateurs significativement ou totalement différents d'une division à l'autre. Compte 
Figure 5 - Utilisation des ICH par les managers opérationnels pour atteindre leurs objectifs

( $\%$ des répondants, $\mathrm{N}=101$ )

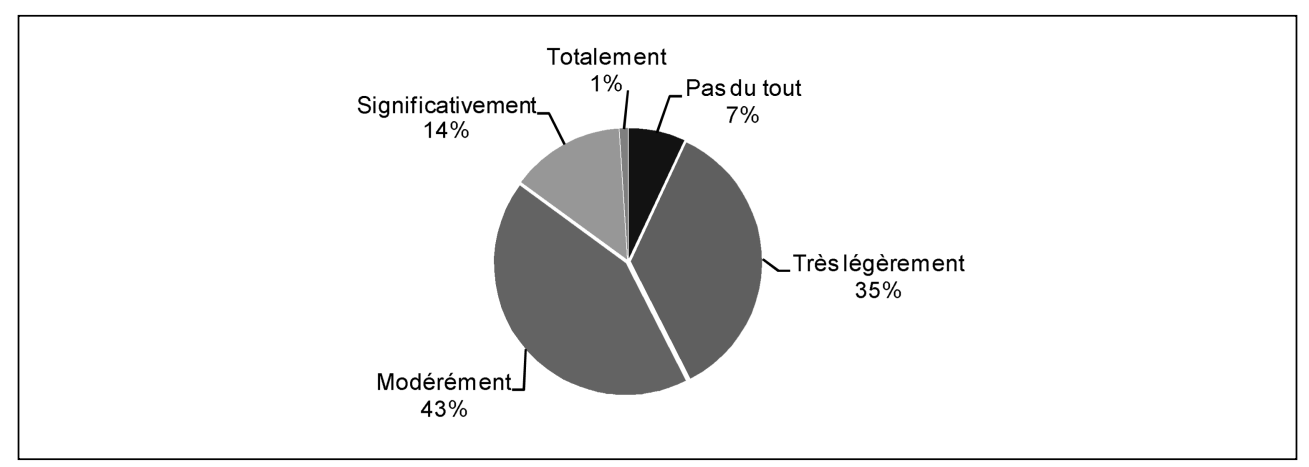

Figure 6 - Degré de différence des ICH entre les divisions

( $\%$ des répondants, $\mathrm{N}=102$ )

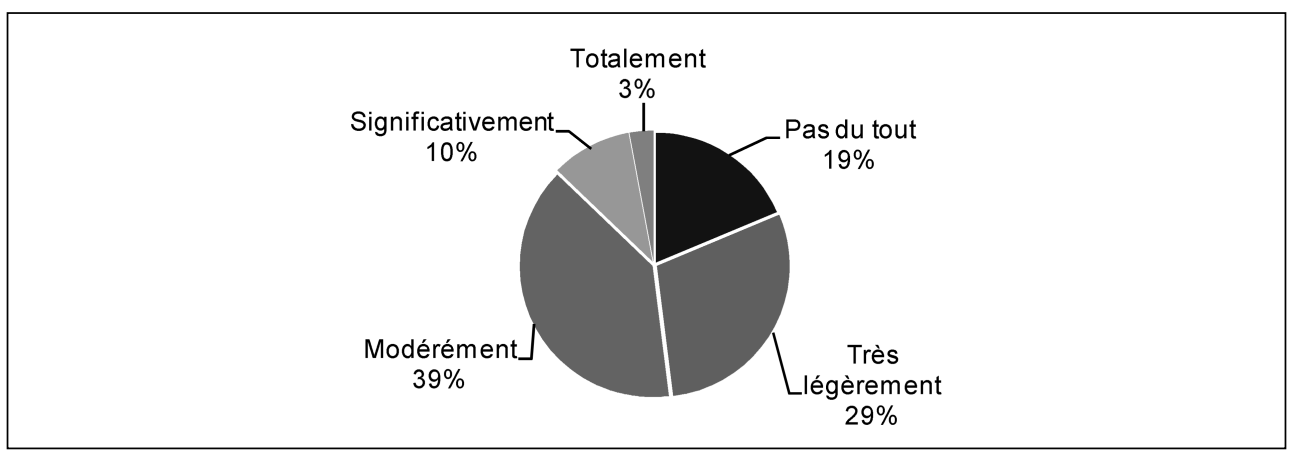

tenu de la grande taille des entreprises qui composent notre échantillon, on peut supposer que ces organisations sont composées de divisions différenciées en termes d'activité, d'objectifs et de compétences clés. L'uniformisation des indicateurs ne permettrait alors pas de satisfaire les besoins spécifiques de chaque division.

Cette remarque nous amène à nous interroger, plus largement, sur la contingence des indicateurs. Plus particulièrement, considé- rant que la mesure de la performance sert à atteindre les objectifs stratégiques, nous pouvons nous intéresser à l'influence de la stratégie sur le choix des indicateurs.

\section{Stratégie et indicateurs de capital humain souhaités}

La littérature a montré que la mise en place d'indicateurs cohérents avec les objectifs stratégiques permet d'améliorer la performance (Ittner et al., 2003; Widener, 2006). 
Quelques efforts ont été développés pour appliquer ce principe d'alignement stratégique aux indicateurs de pilotage de la fonction RH (Becker et al., 2001) ou des attitudes et comportements de l'ensemble des salariés (Huselid et al., 2005).

Notre étude confirme cette hypothèse d'adaptation des indicateurs de capital humain à la stratégie. Dans les entreprises suivant une stratégie de différenciation ${ }^{3}$, les répondants souhaitent en effet des indicateurs de capital humain mesurant les qualités d'innovation des salariés ${ }^{4}$, alors que dans les entreprises cherchant une domination par les coûts, ils préfèrent des indicateurs de capital humain mesurant la capacité des salariés à gérer les coûts ${ }^{5}$. Ainsi, la littérature qui montre que les systèmes de mesure de performance sont en ligne avec la stratégie peut être étendue spécifiquement aux indicateurs de capital humain.

Ces résultats sont illustrés par nos entretiens. Par exemple, un répondant déclare:

" Nous essayons de développer un tableau de bord de capital humain à trois niveaux. Le premier niveau consistera en indicateurs clés basiques concernant toute la force de travail de l'entreprise. Le second niveau sera plus stratégique, liant les indicateurs de capital humain aux plans des business units développés pour l'année suivante. Le troisième niveau sera un effort de mesure du capital humain top-down, centré sur les talents et la gestion de la performance d'un groupe de salariés. Par conséquent, cela nécessite que nous développions un haut niveau d'alignement stratégique pour les deuxièmes et troisièmes niveaux de mesure du capital humain. »

Le rôle que les responsables RH jouent dans la formulation et la mise en place de la stratégie est donc important pour identifier et contribuer à mettre en place les indicateurs pertinents. Qu'en est-il dans notre échantillon?

\section{Rôle de la fonction RH dans la mise en place des indicateurs de capital humain}

Nos résultats montrent que plus les responsables RH participent à la définition de la stratégie, plus l'entreprise est avancée dans la mise en place des indicateurs de capital humain $^{6}$. Les entretiens illustrent cette importance attribuée à la participation à la définition de la stratégie.

«Les responsables RH doivent jouer un rôle plus proactif, stratégique pour identifier les problèmes et recommander les actions qui permettront d'atteindre les objectifs stratégiques. »

"Une scorecard enregistre le score. C'est bien, mais c'est tout ce que cela fait. D'un autre côté, créer et tester un modèle causal montre l'impact des résultats de l'activité,

\footnotetext{
3. La stratégie a été mesurée en demandant aux répondants d'indiquer la part de leur chiffre d'affaires provenant d'activités basées sur une stratégie de différenciation ou de domination par les coûts (le total faisant $100 \%$ ).

Nous avons mesuré l'intérêt pour des ICH alignés à l'aide d'une question listant huit types d'ICH souvent cités dans la littérature. Une analyse en composantes principales a permis d'identifier deux facteurs: un facteur regroupait des indicateurs mesurant la capacité des salariés à réduire ou à gérer les coûts, alors que l'autre facteur était composé d'indicateurs mesurant les capacités entrepreneuriales et d'innovation des salariés.

4. $\mathrm{R}^{2}=0,297, \mathrm{p}<0,01$.

5. $\mathrm{R}^{2}=-0,193, \mathrm{p}<0,05$.

$6 . \mathrm{R}^{2}=0,251, \mathrm{p}<0,01$. Nous avons mesuré l'implication des responsables $\mathrm{RH}$ dans l'élaboration de la stratégie à l'aide d'une question à 6 points (voir les items sur la figure 7).
} 
ce qui vous permet de devenir un vrai partenaire stratégique $»$.

Toutefois, si $76 \%$ des répondants considèrent que la fonction $\mathrm{RH}$ contribue au moins à l'exécution de la stratégie (figure 7), seulement $22 \%$ estiment qu'elle constitue un vrai partenaire dans le développement et la mise en place de la stratégie. Dans notre échantillon, cette faible implication de la fonction RH pourrait être l'une des explications du niveau peu avancé de mise en place d'indicateurs de capital humain.

D'autres explications peuvent néanmoins être trouvées dans les obstacles divers rencontrés dans la mise en place des indicateurs de capital humain. Le tableau 2 montre que si l'adhésion des différentes parties prenantes ne pose pas trop de problèmes, des difficultés sont rencontrées de façon relativement plus importante en ce qui concerne la définition et la démonstration de l'utilité des indicateurs de capital humain, ainsi que sur des aspects purement techniques de traitement de l'information ou d'intégration au sein des opérations de l'organisation.

Certains témoignages confirment ces observations :

« Nous avons besoin de beaucoup de processus [...] Il faut une automatisation pour rendre cela pérenne. Un reporting ad-hoc n'est pas pérenne. »

« Notre service en charge de mesurer le capital humain a changé ses compétences radicalement cette année. Au lieu de cinq juniors spécialistes de technique et de chiffres, nous avons maintenant trois spécialistes de l'analyse et du reporting de données. Nous allons aussi recruter une autre personne pour traduire la stratégie en indicateurs de capital humain. »

«Toutefois, lier les ICH aux autres perspectives dans notre BSC n'est pas aussi simple qu'on pourrait le penser. De plus, si nous

Figure 7 - Rôle de la fonction RH dans la formulation de la stratégie et dans la recommandation d'ICH

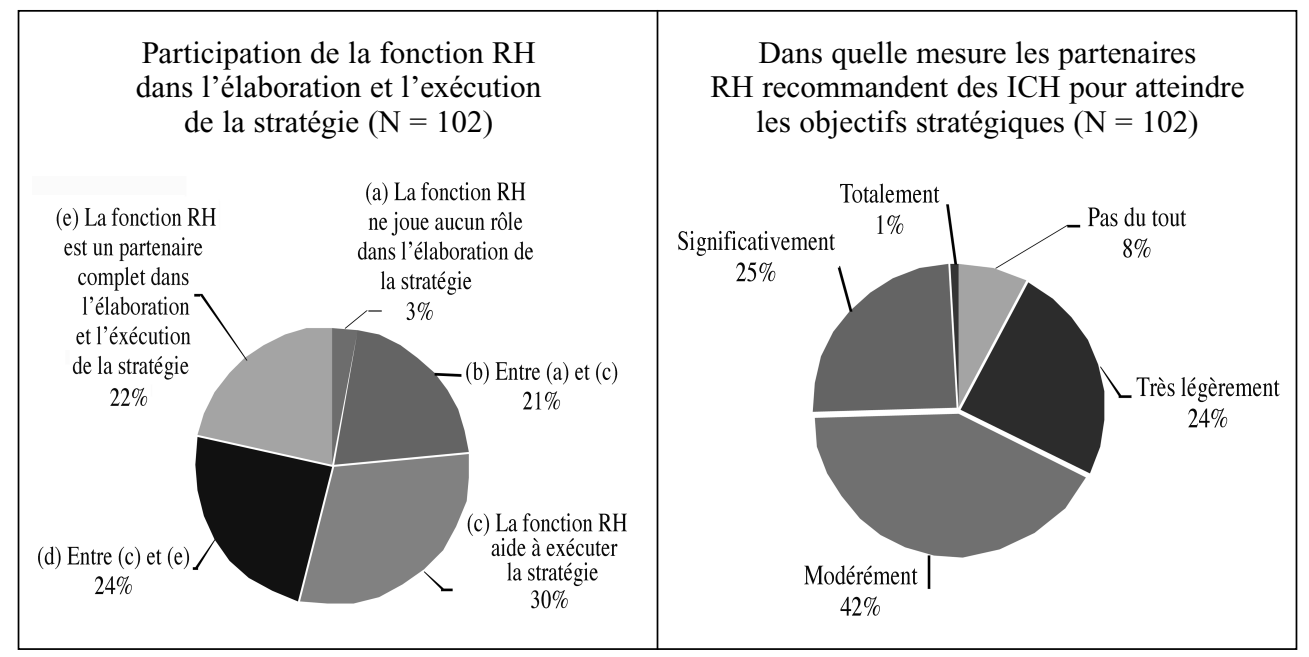


Tableau 2 - Obstacles à la mise en place des ICH (\% des répondants)

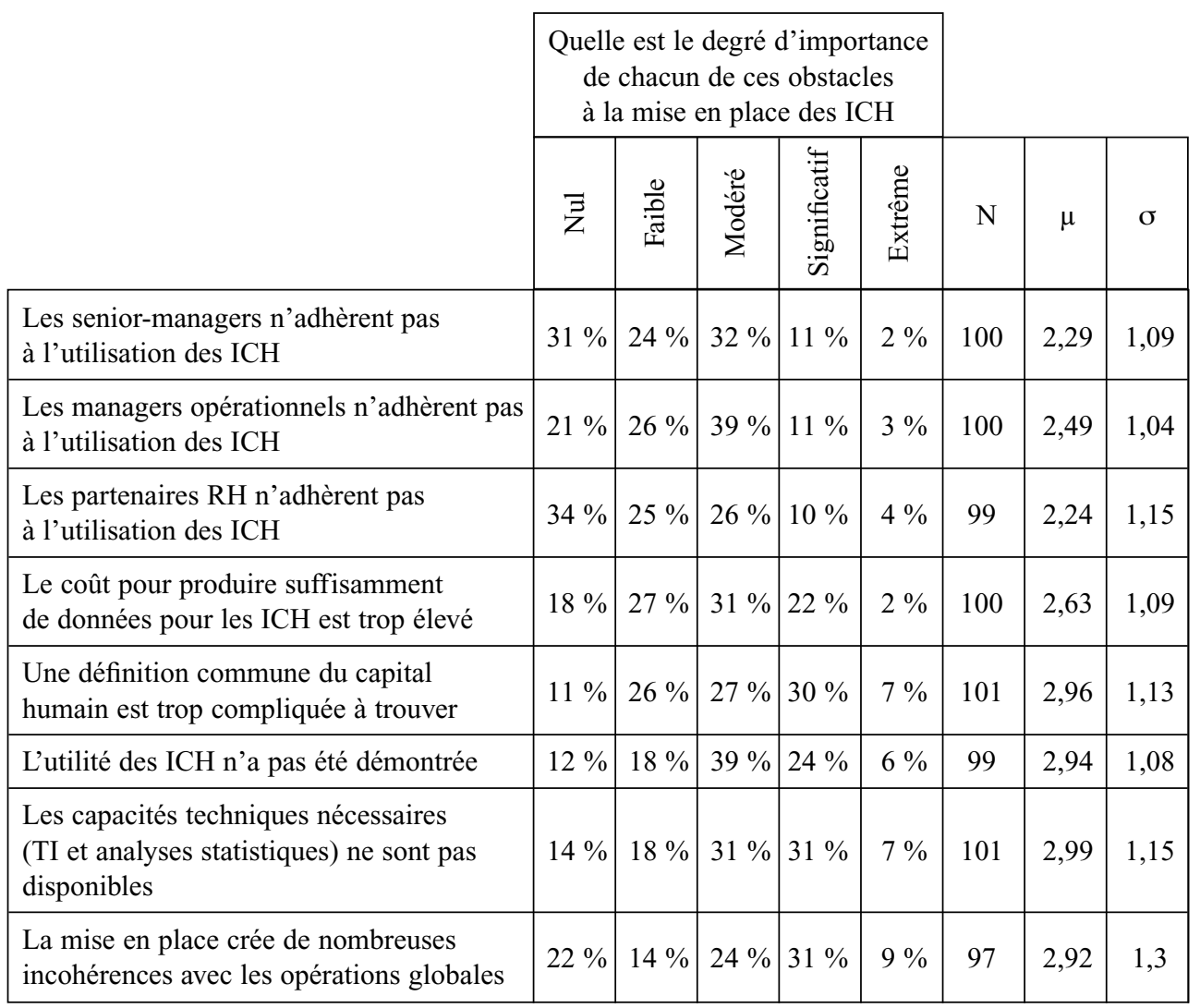

nous concentrons sur les 5 à $10 \%$ des postes stratégiques, nous avons besoin de développer les compétences nécessaires pour mesurer et gérer ces talents. » L'une des solutions qui peut aider le développement des indicateurs de capital humain est de donner aux partenaires $\mathrm{RH}$ les compétences nécessaires, comme l'illustre le commentaire suivant:

« Une possibilité [pour faire face à l'incapacité des managers du capital humain à utiliser les indicateurs pour échanger avec les responsables opérationnels] est de former davantage les partenaires RH qui sont en relation directe avec les divisions. De la sorte, nous pouvons permettre aux partenaires $\mathrm{RH}$ à la fois de mieux connaître l'importance des indicateurs et d'être plus à l'aise pour parler des données pertinentes lorsqu'ils sont avec les responsables de division. »

\section{Conclusion : aider les indicateurs de capital humain à tenir leurs promesses}

Compte tenu du rôle critique joué à la fois par le capital humain et les systèmes de 
mesure de la performance dans la création de valeur et l'atteinte des objectifs stratégiques, le choix et la mise en place d'indicateurs de capital humain a stimulé la recherche dans différentes disciplines de la gestion, en particulier en stratégie, en GRH et en contrôle. Cette étude contribue à cet effort d'interdisciplinarité en analysant les liens entre indicateurs de capital humain, performance et stratégie, y compris le rôle joué par la fonction RH.

Nos résultats montrent que la performance est corrélée avec le niveau de mise en place des indicateurs de capital humain. Les systèmes de mesure du capital humain aident effectivement les organisations à mesurer et à mieux gérer leur performance. Les résultats confirment également le souhait des managers RH de mettre en place des indicateurs de capital humain cohérents avec la stratégie de leur entreprise. Finalement, la participation des responsables RH dans l'élaboration de la stratégie, est associée au niveau de mise en place des ICH, suggérant ainsi que ces professionnels sont bien conscients de l'importance des ICH pour atteindre les objectifs stratégiques.

Toutefois, nos résultats montrent également que la mise en place de ces indicateurs de capital humain est loin d'avoir atteint un stade avancé. Si des efforts sont faits pour développer ces indicateurs dans des systèmes de contrôle intégrés, comprenant tableau de bord et bonus, du che- min reste à parcourir et divers obstacles à surmonter.

Cette étude présente bien sûr des limites. Le cadre d'analyse se concentre sur le capital humain et devrait être élargi aux autres composantes du capital intellectuel. Par ailleurs, l'échantillon est composé de membres du Conference Board: représentativité et validité externe ne sont donc sans doute pas assurées. Enfin, l'utilisation d'une étude en coupe instantanée pour analyser un phénomène qui, par définition, s'étale sur une période relativement longue conduit à interpréter les résultats avec précaution. Ainsi, les corrélations observées ne peuvent pas être interprétées comme des relations de causes à effets.

Malgré ces limites, l'étude confirme à la fois l'intérêt et les difficultés à mettre en place des indicateurs de capital humain pour générer de la performance. Le champ reste donc encore à travailler. En dehors des solutions apportées aux limites méthodologiques évoquées ci-dessus, il serait intéressant de mieux comprendre comment les ICH sont utilisés par les managers non seulement dans leurs décisions opérationnelles, mais également dans l'élaboration de la stratégie. Enfin, la collaboration entre spécialistes en RH et en contrôle, tant professionnels qu'académiques, est plus que jamais nécessaire pour identifier plus facilement les conditions dans lesquelles les indicateurs de capital humain peuvent contribuer à une meilleure performance. 


\section{Bibliographie}

Ashton R.H., "Intellectual capital and value creation: a review", Journal of Accounting Literature, vol. 24, 2005, p. 53-134.

Backhuijs J.B., Holterman W.G., Oudman R.S., Overgoor R.P.M., Zijlstra S.M., "Reporting on intangible assets", in OECD, International Symposium Measuring Reporting Intellectual Capital: Experiences, Issues, and Prospects, OECD, Amsterdam, 1999.

Becker B., Huselid M., Ulrich D., The HR Scorecard: Linking People, Strategy and Performance, Harvard Business School Press, Boston, MA, 2001.

Boudreau J., Ramstad P., "Strategic HRM measurement in the $21^{\text {st }}$ century: from justifying HR to strategic talent leadership", HRM in the $21^{\text {st }}$ Century, M. Goldsmith, R.P. Gandossy, M.S. Efron (Eds.), John Wiley, New York, NY, 2003, p. 79-90.

Davenport T.O., Human capital: What it is and Why People Invest it, Jossey-Bass, San Francisco, CA, 1999.

Eccles R.G., "The performance measurement manifesto", Harvard Business Review, vol. 69, $\mathrm{n}^{\circ} 1,1991$, p. 131-137.

Edvinsson L., "Developing intellectual capital at Skandia", Long Range Planning, vol. 30, $\mathrm{n}^{\circ} 3$, 1997, p. 366-373.

Edvinsson L., Malone M.S., Intellectual capital: realizing your company's true value by finding its hidden roots, HarperCollins, New York, NY, 1997.

Fitz-Enz J., How to Measure Human Resources Management, McGraw-Hill, New York, NY, 1995.

Gates S., Linking People Measures to Strategy, The Conference Board, 1342-03-RR, 2003.

Gates S., Measuring More than Efficiency: The New Role of Human Capital Metrics, The Conference Board, R-1356-04-RR, 2004.

Hamel G., Prahalad C.K., "Competing for the future", Harvard Business Review, vol. 72, $\mathrm{n}^{\circ}$ 4, 1994, p. 122.

Huang T.C., "The effects of linkage between business and human resource management strategies", Personnel Review, vol. 30, n 2, 2001, p. 132-151.

Huselid M.A., Becker B.E., Beatty R.W., The Workforce Scorecard: Managing Human Capital to Execute Strategy, Harvard Business School Press, Boston, MA, 2005.

Ittner C.D., Larcker D.F., "Are nonfinancial measures leading indicators of financial performance? An analysis of customer satisfaction", Journal of Accounting Research, vol. 36, Supplement, 1998, p. 1-35.

Ittner C.D., Larcker D.F., Randall T., "Performance implications of strategic performance measurement in financial services firms", Accounting, Organizations and Society, vol. 28, $\mathrm{n}^{\circ}$ 7-8, 2003, p. 715-741.

Kaplan R.S., Norton D.P., The Balanced Scorecard: Translating Strategy into Action, Harvard Business School Press, Boston, MA, 1996.

Lev B., Intangibles: Management, Measurement, and Reporting, Brookings Institution Press, Washington D.C., 2001. 
Marr B., Gray D., Neely A., "Why do firms measure their intellectual capital?", Journal of Intellectual Capital, vol. 4, $\mathrm{n}^{\circ}$ 4, 2003, p. 441-464.

OECD, International Symposium: Measuring and Reporting Intellectual Capital: Experiences, Issues, and Prospects, OECD, Amsterdam, 1999.

Widener S.K., "Associations between strategic resource importance and performance measure use: the impact on firm performance", Management Accounting Research, vol. $17, \mathrm{n}^{\circ} 4,2006$, p. $433-457$. 
sable de la recherche. Ses recherches portent sur l'évaluation et la valorisation des actifs immatériels et la RSE. Il est vice-président du Réseau international de recherche sur le développement durable (RIODD) et responsable de l'École française de l'immatériel, projet de R\&D de l'Observatoire de l'immatériel.

Redouane EL AMRANI est docteur en sciences de gestion et professeur des systèmes d'information à Reims Management School, France. Il est chercheur associé au LEMNA, université de Nantes. Ses recherches portent sur l'intégration des SI et impacts organisationnels, les centres de compétences ERP et décisionnel et les ERP open source. Il a publié de nombreux articles dans des revues académiques françaises et internationales. Il a présenté également de nombreuses communications dans des actes de conférences internationales.

Jean-Paul FLIPO est professeur émérite de marketing à EM Lyon Business School. Après une carrière dédiée principalement au domaine du marketing des services, pour lequel il a publié de nombreux articles et ouvrages dont le premier en français sur ce sujet, il a évolué vers celui de l'éthique, appliqué plus particulièrement au marketing. Cette évolution a néanmoins commencé depuis longtemps, attestée notamment au travers de la publication d'un certain nombre d'articles plutôt critiques et décalés à propos de cette discipline, et ce depuis 1981, principalement dans la Revue française de gestion. Il collabore avec Fred Seidel non seulement en tant que collègue institutionnel, mais aussi en tant que membre du bureau de l'association EBEN France.
Stephen GATES est professeur de stratégie à Audencia Nantes. Après l'obtention de son doctorat à New York University, Stern School of Business, il a d'abord été analyste financier chez JP Morgan Chase et au Crédit Agricole, puis chercheur principal au sein du "Conference Board », avant de rejoindre le monde académique. Ses thèmes de recherche concernent la mesure du capital humain et les systèmes de mesure de la performance stratégique.

Vanessa KUENTZ est ingénieur de recherche en statistique au sein du Cemagref, Institut de recherche en sciences et technologies pour l'environnement. Elle fait partie de l'équipe Aménités et dynamiques des espaces ruraux dont les recherches portent sur la construction sociale des enjeux environnementaux, la performance environnementale des activités productives, l'analyse des politiques publiques, et l'organisation spatiale des territoires. Elle est titulaire d'une thèse en statistique soutenue à l'université Bordeaux I, intitulée « Contributions à la réduction de dimension $»$. Ses recherches en statistique multidimensionnelle ainsi qu'en régression semi-paramétrique ont donné lieu à des publications dans des revues internationales.

Pascal LANGEVIN est docteur en gestion et enseigne le contrôle de gestion à EM Lyon, en particulier dans les programmes de formation continue. Il a également été gérant d'une petite entreprise industrielle et directeur financier d'une société d'outplacement. Ses principaux travaux de recherche se situent en contrôle organisationnel où il s'intéresse aux effets 
Reproduced with permission of the copyright owner. Further reproduction prohibited without permission. 